\title{
Corollary from the Exact Expression for Enthalpy of Vaporization
}

\author{
A. A. Sobko \\ Department of Physics and Chemistry of New Materials, A. M. Prokhorov Academy of Engineering Sciences, 19 Presnensky Val, \\ Moscow 123557, Russia \\ Correspondence should be addressed to A. A. Sobko, ainrf@mail.ru
}

Received 14 November 2010; Revised 9 March 2011; Accepted 16 March 2011

Academic Editor: K. A. Antonopoulos

Copyright () 2011 A. A. Sobko. This is an open access article distributed under the Creative Commons Attribution License, which permits unrestricted use, distribution, and reproduction in any medium, provided the original work is properly cited.

A problem on determining effective volumes for atoms and molecules becomes actual due to rapidly developing nanotechnologies. In the present study an exact expression for enthalpy of vaporization is obtained, from which an exact expression is derived for effective volumes of atoms and molecules, and under certain assumptions on the form of an atom (molecule) it is possible to find their linear dimensions. The accuracy is only determined by the accuracy of measurements of thermodynamic parameters at the critical point.

\section{Introduction}

In the present study, the relationship is obtained that combines the enthalpy of vaporization with other thermodynamic evaporation parameters from the general expression for the heat of first-order phase transformations. A good agreement of the calculated enthalpy of vaporization with experimental data (within the accuracy of the order of several percent) proves the validity of the assumptions made. By using the obtained expression in the ClausiusClapeyron equation, the relationship between thermodynamic parameters at the critical point is derived, which also well agrees with the experimental data. Also, the expression for determining atomic (ion, molecular) volumes from the thermodynamic parameters is obtained, which, by using geometrical models for atoms and molecules, may predict their linear dimensions.

\section{Background}

First- and second-order phase transitions are widely investigated, mainly in experiments. In the 1930s, L. D. Landau suggested the phenomenological theory of secondorder phase transitions, from which the renormalization group approach and $\varepsilon^{\prime}$-decomposition later were derived. However, by comparing the chapters devoted to first-order phase transitions in the first edition of "Statistical physics,"
1938 [2] with the edition from 1976 [3], we may find them actually similar. we may come to the same conclusion if we compare [2] with recent monograph by Prigogine and Kondepudi "Modern Thermodynamics" [4]. The chapters devoted to first-order phase transitions in both monographs are identical. One may assert that for the last 60-70 years no noticeable progress is observed in the theory of first-order phase transitions. There is a progress in studying critical points which is, however, connected with the fact that at a critical point we have $\Delta V=0, \Delta S=0$, that is, it becomes the second-order transition, theory for which, as was mentioned, is well developed.

The heat of a transition is an important characteristic for first-order phase transitions. In 1762, Black [5] was the first who discovered that water transformation to vapor consumes some heat that he called the latent evaporation heat. Then Black has conducted calorimetric investigations of melting ice.

Despite of longer than 200-year existence of the concept of transition heat we have no analytical expressions (except for empirical) binding the transition heat with other phase transfer parameters. For example, in the fundamental encyclopedia [6] the articles devoted to enthalpy of evaporation, enthalpy of melting, and so on include no formulae but only tables with experimental data. Similar situation is observed with other monographs in which, no expressions are given except for the common definition of transition heat 
TABLE 1: Calculation of evaporation heat.

\begin{tabular}{lccccccccccccc}
\hline Sample & $T$ & $P$ & $V_{L}$ & $\Delta V$ & $r_{a}$ & $V_{a}$ & $V_{\mathrm{fL}}$ & $\sigma$ & $\lambda_{\mathrm{ex}}$ & $\lambda_{T 1}$ & $\delta_{1}$ & $\lambda_{T 2}$ \\
\hline $\mathrm{Ne}$ & 25 & 0,51 & 1,63 & 394 & 1,60 & 1,03 & 0,60 & 5,5 & 1790 & 1549 & 13,5 & 1756 & 1,87 \\
$\mathrm{Ne}$ & 28 & 1,32 & 1,70 & 164 & 1,60 & 1,03 & 0,67 & 4,45 & 1711 & 1449 & 15,3 & 1665 & 2,67 \\
$\mathrm{Ar}$ & 84 & 0,71 & 2,82 & 961 & 1,92 & 1,78 & 1,04 & 11,5 & 6459 & 5377 & 16,7 & 6075 & 5,95 \\
$\mathrm{Ar}$ & 90 & 1,34 & 2,90 & 533 & 1,92 & 1,78 & 1,12 & 10,5 & 6307 & 5186 & 17,8 & 5897 & 6,49 \\
$\mathrm{Kr}$ & 116 & 0,74 & 3,42 & 1259 & 1,98 & 1,96 & 1,46 & 16,1 & 9176 & 7556 & 17,7 & 8373 & 8,75 \\
$\mathrm{Kr}$ & 150 & 6,56 & 3,87 & 164 & 1,98 & 1,96 & 1,91 & 10,0 & 7886 & 6350 & 19,5 & 7214 & 8,52 \\
$\mathrm{Xe}$ & 170 & 1,34 & 4,50 & 1013 & 2,18 & 2,61 & 1,89 & 18,0 & 12446 & 10271 & 17,5 & 11494 & 7,65 \\
$\mathrm{Xe}$ & 216 & 9,19 & 5,10 & 164 & 2,18 & 2,61 & 2,49 & 10,0 & 10642 & 8489 & 20,2 & 9749 & 8,39 \\
$\mathrm{H}_{2}$ & 25 & 3,21 & 3,10 & 48,82 & 1,44 & 0,75 & 2,35 & 1,13 & 836 & 777 & 7,1 & 831 \\
$\mathrm{H}_{2}$ & 32 & 11,0 & 4,25 & 7,88 & 1,44 & 0,75 & 3,50 & 0,11 & 419 & 369 & 11,9 & 404 \\
$\mathrm{~N}_{2}$ & 68 & 0,29 & 3,31 & 1940 & 2,09 & 2,30 & 1,01 & 11,0 & 5822 & 4870 & 16,3 & 5541 & 4,83 \\
$\mathrm{~N}_{2}$ & 90 & 3,36 & 3,75 & 182 & 2,09 & 2,30 & 1,45 & 6,16 & 5057 & 3969 & 21,5 & 4671 & 7,64 \\
$\mathrm{O}_{2}$ & 90 & 0,99 & 2,82 & 724 & 2,00 & 2,02 & 0,80 & 10,0 & 6800 & 5456 & 19,8 & 6393 \\
$\mathrm{O}_{2}$ & 100 & 2,55 & 2,92 & 303 & 2,00 & 2,02 & 0,90 & 7,0 & 6490 & 5049 & 22,2 & 6018 & 7,99 \\
$\mathrm{~F}_{2}$ & 95 & 2,78 & 2,65 & 261 & 2,07 & 2,24 & 0,41 & 10,7 & 6775 & 5030 & 25,8 & 6488 \\
$\mathrm{Cl}_{2}$ & 194 & 0,08 & 4,23 & 19377 & 2,47 & 3,80 & 0,43 & 34,0 & 22161 & 18185 & 17,9 & 21863 \\
$\mathrm{CH}_{4}$ & 100 & 0,34 & 3,64 & 2404 & 2,30 & 3,07 & 0,57 & 15,2 & 8518 & 7395 & 13,2 & 8929 \\
$\mathrm{CH}_{4}$ & 150 & 10,3 & 4,44 & 95,4 & 2,30 & 3,07 & 1,37 & 5,0 & 6656 & 5251 & 21,1 & 6675 \\
\hline
\end{tabular}

$T(\mathrm{~K})$ is the evaporation temperature; $P * 10^{-5}(\mathrm{~Pa})$ is the pressure; $V_{L} * 10^{5}\left(\mathrm{~m}^{3} / \mathrm{mole}\right)$ is the molar volume of liquid; $\Delta V * 10^{5}\left(\mathrm{~m}^{3} / \mathrm{mole}\right)$ is the volume change in evaporation; $r * 10^{10}(\mathrm{~m})$ is the radius of atom (molecule); $\sigma * 10^{3}\left(\mathrm{~J} / \mathrm{m}^{2}\right)$ is the surface tension coefficient; $\lambda_{\text {ex }}(\mathrm{J} / \mathrm{mole}$ ) is the experimental molar evaporation heat; $\lambda_{T 1}(\mathrm{~J} / \mathrm{mole})$ is the molar evaporation heat calculated from the geometrical volume; $V_{\mathrm{Lf}} * 10^{5}\left(\mathrm{~m}^{3} / \mathrm{mole}\right)$ is "free volume" of liquid; $\lambda_{T 2}$ $(\mathrm{J} / \mathrm{mole})$ is the molar evaporation heat calculated from "free volume"; $\delta_{1}$ and $\delta_{2}(\%)$ are errors for $\lambda_{T 1}$ and $\lambda_{T 2}$, respectively.

TABLE 2: Experimental and calculated values for derivatives.

\begin{tabular}{lcccccccc}
\hline Sample & $T_{c}$ & $P_{c}$ & $V_{c}$ & $r_{a}$ & $V_{\text {cf }}$ & $(d P / d T)_{\mathrm{ex}}$ & $(d P / d T)_{T}$ & $\delta$ \\
\hline $\mathrm{Ne}$ & 44,40 & 26,54 & 4,18 & 1,60 & 3,15 & 3,31 & 1,46 \\
$\mathrm{Ar}$ & 150,86 & 48,98 & 7,46 & 1,92 & 5,71 & 1,85 & 1,26 & 1,78 \\
$\mathrm{Kr}$ & 209,39 & 54,97 & 9,20 & 1,98 & 7,24 & 1,48 & 1,41 \\
$\mathrm{Xe}$ & 289,74 & 58,28 & 11,94 & 2,18 & 9,33 & 1,13 & 1,09 \\
$\mathrm{H}_{2}$ & 33,23 & 13,16 & 6,39 & 1,44 & 5,64 & 1,87 & 3,75 \\
$\mathrm{~N}_{2}$ & 126,25 & 33,96 & 8,95 & 2,09 & 6,65 & 1,56 & 1,87 \\
$\mathrm{O}_{2}$ & 154,77 & 50,87 & 7,34 & 2,00 & 5,32 & 1,92 & 1,52 \\
$\mathrm{~F}_{2}$ & 144,00 & 52,20 & 7,10 & 2,07 & 4,88 & 2,25 & 1,89 \\
$\mathrm{Cl}_{2}$ & 417,17 & 77,09 & 12,38 & 2,47 & 8,58 & 1,12 & 2,07 \\
$\mathrm{CH}_{4}$ & 190,55 & 46,41 & 9,88 & 2,30 & 6,81 & 1,62 & 1,15 \\
$\mathrm{NH}_{3}$ & 405,60 & 113,00 & 7,25 & 1,88 & 5,57 & 1,82 & 1,58 \\
$\mathrm{CO}$ & 132,94 & 34,98 & 9,36 & 2,16 & 6,82 & 1,50 & $-3,00$ \\
\hline
\end{tabular}

$T_{c}(\mathrm{~K}), P_{c} * 10^{-5}(\mathrm{~Pa})$, and $V_{c} * 10^{5}\left(\mathrm{~m}^{3} / \mathrm{mole}\right)$ are the critical temperature, pressure, and volume, respectively; $r_{a} * 10^{10}(\mathrm{~m})$ is the atomic $(\mathrm{molecular})$ radius; $V_{\mathrm{cf}} * 10^{5}\left(\mathrm{~m}^{3} / \mathrm{mole}\right)$ is the free critical volume; $(d P / d T)_{\mathrm{ex}} * 10^{-5}$ and $(d P / d T)_{T} * 10^{-5}(\mathrm{~Pa} / \mathrm{K})$ are the experimental and calculated derivatives, respectively; $\delta$ $(\%)$ is the relative error.

$\lambda=T \Delta S$. This is why obtaining expressions that combine the transition heat with other measurable parameters of a firstorder phase transfer would be a substantial contribution into the first-order phase transformations. In monographs $[1,7]$ a number of expressions are suggested for calculating the enthalpy of evaporation, for example, the Douglas-Avakyan method: $\Delta H_{v}=T \Delta V_{v}\left(397 \sum n P / 1512 \delta_{T}-7 \sum n^{3} P / 216 \delta_{T}\right)$; the Riedel method: $\Delta H_{v_{b}}=1.093 R T_{c}\left(T_{b_{r}}\left(\ln P_{c}-1\right) /(0.930-\right.$ $\left.T_{b_{r}}\right)$ ); the Chen method: $\Delta H_{v_{b}}=R T T_{b_{r}}\left(\left(3.978 T_{b_{r}}-3.938+\right.\right.$ $\left.1.555 \ln P_{c}\right) /\left(1.07-T_{b_{r}}\right)$.
These methods help in the calculation of the enthalpy of evaporation for various complex organic substances with a high accuracy. As shown in [1, Table 6.3], the accuracy is several percent, and in particular, for inert gases the accuracy is $2.1-2.5 \%$. In engineering calculations this is rather satisfactory. But, as P. Dirac noted "Physical law should have mathematical beauty," which is not the case of the presented expressions. Presently the author is developing the model described below for calculating complex organic compounds. 
TABLE 3: Volumes and radii of molecules in spherical model.

\begin{tabular}{lcccccccccc}
\hline Sample & $T_{c}$ & $P_{c}$ & $V_{c}$ & $(d P / d T)_{c}$ & $V_{0}$ & $r_{a}$ & $r_{T}$ & $\delta_{1}$ & $r_{W}$ & $\delta_{2}$ \\
\hline $\mathrm{Ne}$ & 44,400 & 26,54 & 4,18 & 3,31 & 18,47 & 1,60 & 1,64 & 2,51 & 1,77 \\
$\mathrm{Ar}$ & 150,86 & 48,98 & 7,45 & 1,85 & 33,22 & 1,92 & 1,99 & 3,89 & 2,14 & 11,7 \\
$\mathrm{Kr}$ & 209,39 & 54,97 & 9,20 & 1,48 & 39,61 & 1,98 & 2,11 & 6,82 & 2,30 & 16,2 \\
$\mathrm{Xe}$ & 289,74 & 58,28 & 11,83 & 1,13 & 48,23 & 2,18 & 2,26 & 3,60 & 2,50 & 14,7 \\
$\mathrm{NH}_{3}$ & 405,6 & 113,0 & 7,25 & 1,82 & 30,92 & 1,82 & 1,95 & 6,92 & 2,12 & 16,6 \\
$\mathrm{CH}_{4}$ & 190,55 & 46,41 & 9,90 & 1,34 & 38,87 & 2,00 & 2,10 & 5,08 & 2,36 \\
$\mathrm{Li}$ & 3800 & 968,3 & 6,94 & 1,62 & 13,87 & 1,55 & 1,49 & 3,82 & 2,09 & 35,1 \\
\hline
\end{tabular}

$T_{c}$ is the critical temperature $(\mathrm{K}), P_{c} * 10^{-5}$ is the critical pressure $(\mathrm{Pa}), V_{c} * 10^{5}$ is the critical (geometric) volume $\left(\mathrm{m}^{3} / \mathrm{mole}\right),(d P / d T)_{c} * 10^{-5}(\mathrm{~Pa} / \mathrm{K})$ is taken in a critical point, $V_{0}$ is the effective molecule volume, $r_{a} * 10^{10}(\mathrm{~m})$ are the molecule radii taken from publications $[1], r_{T} * 10^{10}(\mathrm{~m})$ are the calculated molecule radii, $r_{W} * 10^{10}(\mathrm{~m})$ are the molecule radii obtained from Van der Waals equation, and $\delta_{1}$ and $\delta_{2}$ are the errors for $r_{T}$ and $r_{W}$, respectively.

TABLE 4: Volumes and linear dimensions for twoatomic molecules in ellipsoidal model.

\begin{tabular}{lcccccccc}
\hline Sample & $T_{c}$ & $P_{c}$ & $V_{c}$ & $(d P / d T)_{c}$ & $2 c$ & $V_{0}$ & $A$ \\
\hline $\mathrm{H}_{2}$ & 33.23 & 13.16 & 6.39 & 1.87 & 0.74 & 12.49 & 1.47 \\
$\mathrm{~N}_{2}$ & 126.25 & 33.96 & 8.95 & 1.56 & 1.10 & 41.75 & 2.20 \\
$\mathrm{O}_{2}$ & 154.77 & 50.87 & 7.34 & 1.92 & 1.21 & 35.18 & 2.09 & 2.13 \\
$\mathrm{~F}_{2}$ & 144.00 & 52.20 & 6.62 & 2.25 & 1.42 & 36.83 & 2.15 & 2.01 \\
$\mathrm{Cl}_{2}$ & 417.17 & 77.09 & 12.38 & 1.12 & 1.99 & 58.04 & 2.54 \\
$\mathrm{CO}$ & 132.94 & 34.98 & 9.36 & 1.50 & 1.13 & 43.88 & 2.24 \\
\hline
\end{tabular}

$T_{c}$ is the critical temperatrue $(\mathrm{K}), P_{c} * 10^{-5}$ is the critical pressure $(\mathrm{Pa}), V_{c} * 10^{5}$ is the critical (geometrical) volume $\left(\mathrm{m}^{3} / \mathrm{mole}\right),(d P / d T)_{c} * 10^{-5}(\mathrm{~Pa} / \mathrm{K})$ is taken at the critical point, $V_{0} * 10^{30}\left(\mathrm{~m}^{3}\right)$ is the calculated inaccessible molecule volume, $2 c * 10^{10}(\mathrm{~m})$ is the distance between the atoms in the molecule, and $a * 10^{10}$ and $b * 10^{10}(\mathrm{~m})$ are the major and minor semiaxes of ellipsoid.

\section{Derivation of the Enthalpy of Evaporation and Comparison with Experimental Data}

In [8], the general expression for the heat of first-order phase transitions was derived from the first law of thermodynamics

$$
\lambda=T \int_{v_{1}}^{v_{2}}\left(\frac{\partial S}{\partial V}\right)_{T} d V+\Delta A, \quad \text { or } \quad \lambda=T \Delta_{T} S+\Delta A,
$$

where $T$ is the transition temperature, $\Delta A$ is the work performed by the system, and $\int_{V_{1}}^{V_{2}}(\partial S / \partial V)_{T} d V, \Delta_{T} S$ is the entropy variation for the phase transition at constant temperature.

Numerical calculations require that $S(V)$ is found. The entropy is calculated from the general definition [3]: $S=$ $k \ln V_{\mathrm{ph}} /(2 \pi \hbar)^{s}$ and $\Delta S=k \ln \left(V_{\mathrm{ph}}\right)_{n} /\left(V_{\mathrm{ph}}\right)_{o}$, where $k$ is the Boltzmann constant, $V_{\mathrm{ph}}$ is the volume of phase space occupied by the system, $s$ is the number of degrees of freedom, $\Delta S$ is the entropy change in the phase transition, $\left(V_{\mathrm{ph}}\right)_{n}$ is the phase volume of a new phase, and $\left(V_{\mathrm{ph}}\right)_{0}$ is the phase volume for the previous phase. form

Thus, the heat of first-order phase transition takes the

$$
\lambda=k T \ln \frac{\left(V_{\mathrm{ph}}\right)_{n}}{\left(V_{\mathrm{ph}}\right)_{o}}+\Delta A .
$$

The volumes of phase spaces and expressions for the work are specified for each particular phase transition. In the present paper all calculations are made per one mole of substance, hence, all extensive parameters refer to one mole.
In [9], the approximation method is suggested for calculating the entropy from the general expression $S=$ $k \ln V_{\mathrm{ph}} /(2 \pi \hbar)^{N}$, where $V_{\mathrm{ph}}$ is the volume of the phase space for the system.

For liquid and gas states the system energy has the form

$$
\begin{aligned}
\frac{p_{x 1}^{2}}{2 m_{1}} & +\frac{p_{y 1}^{2}}{2 m_{1}}+\frac{p_{1 z}^{2}}{2 m_{1}}+\cdots+\frac{p_{z N}{ }^{2}}{2 m_{N}} \\
& +U\left(\mathbf{r}_{1}, \ldots, \mathbf{r}_{n}\right)=E, \quad 1 \leq n \leq N_{A} .
\end{aligned}
$$

Since we deal with one mole of single-component sample all the masses are equal and $N=N_{A}$ is the Avogadro constant so that the volume of the phase space is $V_{\mathrm{ph}}=$ $\iint_{6 N} \prod_{n=1}^{N_{A}} d^{3} p_{n} d^{3} r_{n}$.

Equation (3) can be written in the form $\sum_{\text {in }} p_{\text {in }}^{2}=$ $\left(2 m\left(E-U\left(\mathbf{r}_{1}, \ldots, \mathbf{r}_{n}\right)\right)\right)$, which describes a $3 \mathrm{~N}$-dimension sphere in $\mathbf{p}$-space. Hence, the $3 \mathrm{~N}$-dimensional integral over pulses $\mathbf{p}$ is equal to the volume of the sphere of radius $\left(2 m\left(E-U\left(\mathbf{r}_{1}, \ldots, \mathbf{r}_{n}\right)\right)\right)^{1 / 2}$, and the expression for the phase space volume takes the form

$$
\iint_{3 N} \frac{\pi^{3 N / 2}}{(3 N / 2) !}\left(2 m\left(E-U\left(r_{1}, \ldots r_{n}\right)\right)\right)^{3 N / 2} \prod_{n=1}^{N_{A}} d^{3} r .
$$

The integration results in a reduction of the dimension from $6 N$ to $3 N$. Nevertheless, it is impossible to take the rest integral without additional assumptions. To approximately take the needed $3 \mathrm{~N}$-dimensional integral we will consider the behavior of the distribution function for the kinetic energy of particles near the point of phase transition. Not specifying 
TABLE 5: Calculation results for evaporation heat.

\begin{tabular}{lccccccccccccc}
\hline Sample & $T$ & $P$ & $V_{L}$ & $\Delta V$ & $r_{a}$ & $V_{a}$ & $V_{\mathrm{fL}}$ & $\sigma$ & $\lambda_{\mathrm{ex}}$ & $\lambda_{T 1}$ & $\delta_{1}$ & $\lambda_{T 2}$ & $\delta_{2}$ \\
\hline $\mathrm{Ne}$ & 25 & 0,51 & 1,63 & 394 & 1,64 & 1,11 & 0,52 & 5,5 & 1790 & 1757 & 1,84 & 1796 & 0,36 \\
$\mathrm{Ne}$ & 28 & 1,32 & 1,70 & 164 & 1,64 & 1,11 & 0,59 & 4,45 & 1712 & 1665 & 2,75 & 1703 & 0,52 \\
$\mathrm{Ar}$ & 84 & 0,71 & 2,82 & 961 & 2,00 & 1,99 & 0,83 & 11,5 & 6459 & 6070 & 6,02 & 6272 & 2,89 \\
$\mathrm{Ar}$ & 90 & 1,34 & 2,90 & 533 & 2,00 & 1,99 & 0,91 & 10,5 & 6307 & 5895 & 6,53 & 6089 & 3,46 \\
$\mathrm{Kr}$ & 116 & 0,74 & 3,42 & 1259 & 2,11 & 2,37 & 1,05 & 16,1 & 9176 & 8377 & 8,71 & 8816 & 3,92 \\
$\mathrm{Kr}$ & 150 & 6,56 & 3,87 & 164 & 2,11 & 2,37 & 1,50 & 10,0 & 7886 & 7220 & 8,44 & 7590 & 3,75 \\
$\mathrm{Xe}$ & 170 & 1,34 & 4,50 & 1013 & 2,28 & 2,91 & 1,59 & 18,0 & 12446 & 11491 & 7,67 & 11830 & 4,95 \\
$\mathrm{Xe}$ & 216 & 9,19 & 5,10 & 164 & 2,28 & 2,91 & 2,19 & 10,0 & 10642 & 9740 & 8,48 & 10027 & 5,78 \\
$\mathrm{H}_{2}$ & 25 & 3,21 & 3,10 & 48,82 & 1,45 & 0,77 & 2,33 & 1,13 & 836 & 831 & 0,60 & 833 & 0,34 \\
$\mathrm{H}_{2}$ & 32 & 11,0 & 4,25 & 7,88 & 1,45 & 0,77 & 3,48 & 0,11 & 419 & 404 & 3,58 & 405 & 3,44 \\
$\mathrm{~N}_{2}$ & 68 & 0,29 & 3,31 & 1940 & 2,23 & 2,54 & 0,77 & 11,0 & 5822 & 5533 & 4,96 & 5742 & 1,38 \\
$\mathrm{~N}_{2}$ & 90 & 3,60 & 3,75 & 182 & 2,23 & 2,54 & 1,21 & 6,16 & 5057 & 4668 & 7,69 & 4832 & 4,46 \\
$\mathrm{O}_{2}$ & 90 & 0,99 & 2,82 & 724 & 2,16 & 2,17 & 0,65 & 10,0 & 6800 & 6400 & 5,88 & 6583 & 3,19 \\
$\mathrm{O}_{2}$ & 100 & 2,55 & 2,92 & 303 & 2,16 & 2,17 & 0,78 & 7,0 & 6490 & 5992 & 7,67 & 6163 & 5,04 \\
$\mathrm{~F}_{2}$ & 95 & 2,78 & 2,65 & 261 & 2,22 & 2,30 & 0,35 & 10,7 & 6775 & 6491 & 4,19 & 6636 & 2,04 \\
$\mathrm{Cl}_{2}$ & 194 & 0,08 & 4,23 & 19377 & 2,44 & 3,66 & 0,57 & 34,0 & 22161 & 21925 & 1,06 & 21346 & 3,68 \\
$\mathrm{CH}_{4}$ & 150 & 10,3 & 4,44 & 95,4 & 2,48 & 2,33 & 2,11 & 5,0 & 6656 & 6674 & 0,27 & 6087 & 8,55 \\
\hline
\end{tabular}

$T(\mathrm{~K})$ is the evaporation temperature, $P * 10^{-5}(\mathrm{~Pa})$ is the pressure, $V_{L} * 10^{5}\left(\mathrm{~m}^{3} / \mathrm{mole}\right)$ is the volume of liquid, $\Delta V * 10^{5}\left(\mathrm{~m}^{3} / \mathrm{mole}\right)$ is the volume change in evaporation, $r_{a} * 10^{10}$ is the radius of atom (molecule), $V_{a} * 10^{5}\left(\mathrm{~m}^{3} / \mathrm{mole}\right)$ is the volume occupied by atoms (molecules), $V_{\mathrm{fL}} * 10^{5}\left(\mathrm{~m}^{3} / \mathrm{mole}\right)$ is the free volume of liquid, $\sigma * 10^{3}\left(\mathrm{~J} / \mathrm{m}^{2}\right)$ is the surface tension coefficient, $\lambda_{\mathrm{ex}}(\mathrm{J} / \mathrm{mole})$ is the experimental value of evaporation heat, $\lambda_{T 1}(\mathrm{~J} / \mathrm{mole})$ is the evaporation heat calculated from reference data on volume and size of atoms (molecules), $\lambda_{T 2}(\mathrm{~J} / \mathrm{mole})$ is the evaporation heat calculated from data on volumes and dimensions of atoms (molecules) obtained in the present paper, and $\delta_{1}$ and $\delta_{2}(\%)$ are relative errors.

the particular distribution function we may assert that it has a bell shape with the maximum that moves right with temperature. Near the phase transition point such evolution of the distribution function is impossible because the system temperature is constant and the heat still passes to the system. The only possible variation of the distribution function in this case is its narrowing and transformation to the delta function in the limit $T \rightarrow T_{\mathrm{ph}}$. In this case the most probable and the mean values of the kinetic energy coincide. This is not a rigorous proof for narrowing the distribution function. However, we can make the basic assumption, which is confirmed by satisfactory agreement between experimental and calculation results: most of atoms (molecules, ions) near the first-order phase transition are in the state with a mean kinetic energy.

Since $\left(E-U\left(\mathbf{r}_{1}, \ldots, \mathbf{r}_{n}\right)\right)$ is the system kinetic energy we, according to the theorem on equal distribution of kinetic energy over degrees of freedom [10], can substitute it with the mean value $3 N_{A} k T / 2=3 R T / 2$, where $R=k N_{A}$ is the absolute gas constant. Then,

$$
\begin{aligned}
V_{\mathrm{ph}} & =\iint_{3 N} \frac{\pi^{3 N / 2}}{(3 N / 2) !}\left(\frac{3}{2} 2 m R T\right)^{3 N / 2} \prod_{n=1}^{N_{A}} d^{3} r_{n} \\
& =\frac{\pi^{3 N / 2}}{(3 N / 2) !}(2 m R T)^{3 N / 2} V^{N} .
\end{aligned}
$$

Hence, the volume of the phase space has the form:

$$
\begin{aligned}
V_{\mathrm{ph} L} & =\frac{(3 \pi m R T)^{3 N / 2}}{(3 N / 2) !} V_{L}^{N}, \\
V_{\mathrm{ph} G} & =\frac{(3 \pi m R T)^{3 N / 2}}{(3 N / 2) !} V_{G}^{N},
\end{aligned}
$$

where $V_{L}$ and $V_{G}$ are the volumes of liquid and gas, respectively. The expression for the evaporation heat at the saturation curve can be found from general expression (2). One can see from (6) and (7) that the expressions for the volumes of phase spaces coincide for liquid and gas. One can easily write the expression for the entropy variation in this case:

$$
\begin{gathered}
k \ln \left(\frac{(2 \pi m R T)^{3 N / 2}}{(3 N / 2) !} V^{N}\right), \\
\left(\frac{\partial S}{\partial V}\right)_{T}=\frac{k N}{V}=\frac{R}{V}, \\
T \int_{v_{1}}^{V_{2}} \frac{R}{V} d V=R T \ln \frac{V_{G}}{V_{L}}=R T \ln \left(1+\frac{\Delta V}{V_{L}}\right),
\end{gathered}
$$

where $\Delta V$ is the volume change, $V_{L}$ is the volume of liquid, and $V_{G}$ is that of gas.

Work on volume enlarging is $A_{1}=P \Delta V$. In the liquidgas transfer in addition to the work on volume enlarging the work is performed against surface tension force $A_{2}=\sigma F N_{1}$, where $\sigma$ is the surface tension coefficient, $F$ is the surface of liquid, $N_{1}=V_{a} / F d$ is the number of single-molecular layers, $V_{a}$ is the volume occupied by atoms (molecules, ions), $d$ is the width of single-molecular layer that in the present paper is $r, r$ is radius of atom (molecule, ion), and $\alpha=1.717$ is the packing index.

Hence, $A_{2}=\sigma F N_{1}=\sigma F V_{a} / F d=\sigma V_{a} / \alpha r$.

The expression for evaporation heat along the saturation curve has the form

$$
\lambda=R T \ln \left(1+\frac{\Delta V}{V_{L}}\right)+P \Delta V+\frac{\sigma V_{a}}{\alpha r} .
$$




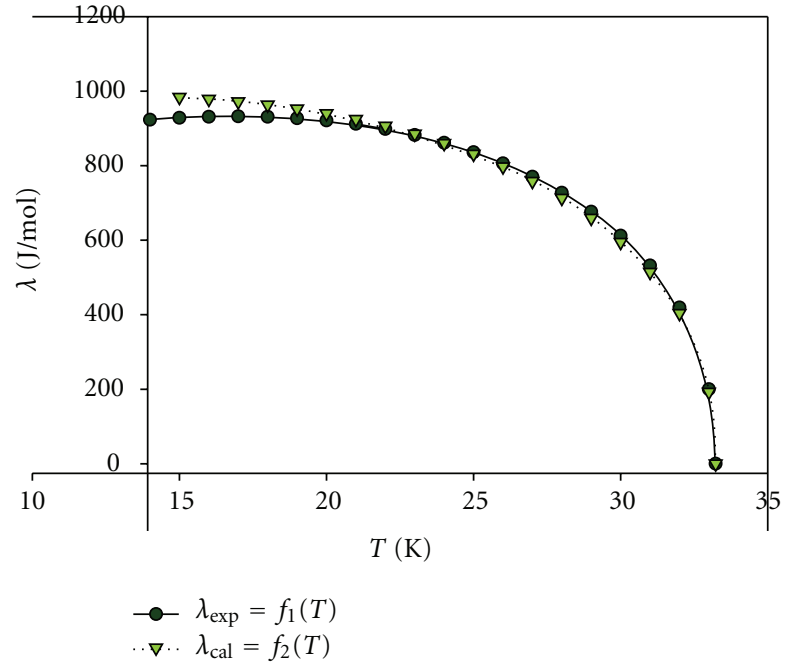

FIgURE 1: Temperature dependence of the experimental enthalpy of evaporation $\lambda_{\exp }=f_{1}(T)$ and calculated enthalpy of evaporation $\lambda_{\text {cal }}=f_{2}(T)$ for hydrogen.

It is not clear which volume of liquid phase $V_{L}$ should be used in the calculations, the geometrical volume or "free volume" $V_{f L}=V_{L}-V_{a}$, where $V_{a}$ is the volume occupied by atoms (molecules, ions). This is why the calculations are performed for both the geometrical (experimental) volume and "free volume." The experimental data on saturation curve were taken from [11], and atomic radii were used from [12]. For the radii of twoatomic molecules we took half the distance between the centers of nuclei [13] plus Van der Waals radii [12]. The results are presented in Table 1.

One can see that the evaporation heat calculated by using the "free volume" noticeably better agrees with the experimental data, hence, the expression for the evaporation heat can be finally written in the form

$$
\lambda=R T \ln \left(1+\frac{\Delta V}{V_{\mathrm{fL}}}\right)+P \Delta V+\frac{\sigma V_{a}}{\alpha r_{a}} .
$$

Expression (10) includes the term with a surface tension coefficient. A contribution of the term into the enthalpy of evaporation is on the order of $10 \%$. Unfortunately, the author has no data on the surface tension coefficient in the interval from the triple to boiling point for most of substances, and the results for only several values of the enthalpy of evaporation are given in the table. For hydrogen, the surface tension coefficient is known over the whole interval from the triple to boiling point, which gives a possibility to draw a temperature dependence for the enthalpy of evaporation. The result is shown in Figure 1.

As one can see, the experimental values of enthalpy of evaporation well agree with those calculated by formula (10). A distinction at low temperatures is explained by that at those temperatures the calculation of the phase space volume should take into account quantum corrections.

Note that, in [14], the method used above gives a good agreement between calculated and experimental values for the enthalpy of fusion.

\section{Conclusion and Test of the Relationship Binding Critical Parameters}

The Clausius-Clapeyron equation

$$
\frac{d P}{d T}=\frac{\Delta S}{\Delta V}=\frac{\lambda}{T \Delta V}
$$

is rigorously derived from thermodynamic relationships and experimentally verified. Expression (10) for the evaporation heat is also sufficiently rigorously obtained, and the calculation results agree with the experimental data. Hence, by substituting (10) into (11) and passing to the critical point limit $\Delta V \rightarrow 0$ we obtain the relationship between the thermodynamic parameters at a critical point

$$
\begin{gathered}
\frac{d P}{d T}=\frac{R T}{T \Delta V} \ln \left(1+\frac{\Delta V}{V_{\mathrm{fL}}}\right)+\frac{P \Delta V}{T \Delta V}+\frac{\sigma V_{a}}{\alpha r_{a} T \Delta V}, \\
=\frac{R}{\Delta V} \ln \left(1+\frac{\Delta V}{V_{\mathrm{fL}}}\right)+\frac{P}{T}+\frac{\sigma V_{a}}{\alpha r_{a} T \Delta V}, \\
\lim _{\Delta V \rightarrow 0} \frac{d P}{d T}=\lim _{\Delta V \rightarrow 0} \frac{R}{\Delta V} \ln \left(1+\frac{\Delta V}{V_{\mathrm{fL}}}\right)+\lim _{\Delta V \rightarrow 0} \frac{P}{T} \\
+\lim _{\Delta V \rightarrow 0} \frac{\sigma V_{a}}{\alpha r_{a} T \Delta V}, \\
\lim _{\Delta V \rightarrow 0} \frac{d P}{d T}=\left(\frac{d P}{d T}\right)_{c}, \lim _{\Delta V \rightarrow 0} \frac{P}{T}=\frac{P_{c}}{T_{c}}, \\
\lim _{\Delta V \rightarrow 0} \frac{R}{\Delta V} \ln \left(1+\frac{\Delta V}{V_{\mathrm{fL}}}\right)=\lim _{\Delta V \rightarrow 0} \frac{R}{\Delta V} \frac{\Delta V}{V_{\mathrm{fL}}}=\lim _{\Delta V \rightarrow 0} \frac{R}{V_{\mathrm{fL}}}=\frac{R}{V_{\mathrm{flc}}}, \\
\lim _{\Delta V \rightarrow 0} \frac{\sigma V_{a}}{\alpha r_{a} T \Delta V}=0 .
\end{gathered}
$$

The latter statement can be proved. As was shown [15] near the critical point we have $\sigma \sim \sigma_{0}\left(1-T / T_{c}\right)^{3 / 2}$ and (see [4]) $\Delta V \sim\left(1-\left(T / T_{c}\right)\right)^{\beta}$, where the theoretical value is $\beta=$ $1 / 2$ and experimental values are $\beta=0.3-0.4$. Thus, we have

$$
\lim _{\Delta V \rightarrow 0} \frac{\sigma V_{a}}{\alpha r_{a} T \Delta V}=\frac{\sigma_{0} V_{a}}{\alpha r_{a}} \lim _{T \rightarrow T_{c}} \frac{\left(1-T / T_{c}\right)^{3 / 2}}{T\left(1-T / T_{c}\right)^{\beta}}=0 .
$$

At the critical point the following relationship should hold:

$$
\left(\frac{d P}{d T}\right)_{c}=\frac{R}{V_{\mathrm{fL} c}}+\frac{P_{c}}{T_{c}}
$$

Subscript $c$ indicates that all the thermodynamic parameters are taken at the critical point.

Formula (14) is rigorously obtained from the exact Clausius-Clapeyron equation and (10) that is confirmed by a good description for the evaporation heat. However, it is worth verifying for particular substances. We can calculate the derivative $(d P / d T)_{c, T}$ from experimental data [11-13] by using formula (14) and from expression $(d P / d T)_{c, \text { ex }}=\left(P_{c}-\right.$ $\left.P_{1}\right) /\left(T_{c}-T_{1}\right)$, where $P_{1}, T_{1}$ are experimental pressure and temperature closest to the critical point on the equilibrium curve. A good agreement between the derivatives $(d P / d T)_{c, T}$ 
and $(d P / d T)_{c, \text { ex }}$ will be an argument for the validity of (14). The calculation results are presented in Table 2.

Small deviations in the values of derivatives (on the order of several percent) are explained by an insufficient measurement accuracy near the critical point and by approximate character for values of molecule radii. For example, the values of atomic radii given in [16] and calculated by eight various authors differ by several percent and even by 10 or more percent.

\section{Definition of Volumes and the Linear Sizes of Atoms and Molecules}

Formula (14) whose validity was rigorously proved and experimentally verified includes the "free" system critical volume $V_{\mathrm{fc}}=V_{c}-N_{\mathrm{A}} V_{0}$, where $V_{c}$ is the experimental value for the critical volume, $N_{A}=6.02 * 10^{23}$ is the Avogadro constant, and $V_{0}$ is the volume of atom (molecule, ion). Hence, one can obtain the following formula for determining the effective volumes of atoms (molecules, ions) via the thermodynamic parameters at a critical point:

$$
V_{0}=\frac{1}{N_{A}}\left\{V_{c}-\frac{R}{(d P / d T)_{c}-P_{c} / T_{c}}\right\} .
$$

By using geometrical models for atoms (molecules) one can calculate their linear parameters.

5.1. Spherical Model. Spherical model is adequate to noble gases, alkali metal ions, $\mathrm{CH}_{4}$ and $\mathrm{NH}_{3}$ molecules due to smallness of hydrogen atoms. The corresponding atomic (molecular) radii can be found by using (15) from the relationship $V_{0}=(4 / 3) \pi r^{3}$ or $r=\sqrt[3]{3 V_{0} / 4 \pi}$. The constant $b=V_{c} / 3$ in Van der Waals equation is treated as the volume occupied by molecules, and the effective radii in the spherical Van der Waals model can be calculated by the formula $r_{W}=$ $\sqrt[3]{V_{c} / 4 \pi N_{A}}$. These results are presented in Table 3 .

One can see from Table 3 that the results calculated by formula (15) well agree with the reference data; however, they are more reliable because most of methods for determining molecule dimensions are approximate whereas the method suggested is accurate and its accuracy is only determined by the measurement accuracy for the thermodynamic parameters at a critical point.

5.2. Ellipsoidal Model. For twoatomic molecules that are symmetric and for those combined from the atoms of close dimensions the ellipsoid of revolution can be suggested as the geometrical model:

$$
\frac{x^{2}}{a^{2}}+\frac{y^{2}}{b^{2}}+\frac{z^{2}}{b^{2}}=1
$$

The volume of the ellipsoid of revolution is $V_{0}=$ $(4 / 3) \pi a b^{2}=(4 / 3) \pi\left(a^{2}-c^{2}\right) a$, where $2 c$ is the distance between the atomic centers taken from [13]. The volume $V_{0}$ is calculated by formula (14). Thus, the equation for finding the major semiaxis takes the form

$$
a^{3}-c^{2} a-\frac{3 V_{0}}{4 \pi}=0 .
$$

As expected, the equation parameters are so that there is one real solution and two complex solutions. The equation assumes an analytical solution [17], namely,

$\sin (\omega)=-\frac{8 \pi c^{3}}{9 \sqrt{3} V_{0}}, \quad \operatorname{tg}(\varphi)=\sqrt[3]{\operatorname{tg}\left(\frac{\omega}{2}\right)}, \quad a=-\frac{2 c}{\sqrt{3} \sin (2 \varphi)}$.

By using $a$ one can find $b=\sqrt{a^{2}-c^{2}}$. The calculation results are given in Table 4 .

For the model of twoatomic molecules one can also suggest the body of revolution formed by the Cassinian oval:

$$
\left(x^{2}+y^{2}\right)^{2}-2 c^{2}\left(x^{2}-y^{2}\right)-\left(d^{4}-c^{4}\right)=0,
$$

where $d^{2}$ is the product of the distances from the centers to an arbitrary point on the curve, and $2 c$ is the distance between the atomic centers. The obtained body of revolution, in contrast to an ellipsoid that has a convex surface, may have a dumbbell shape with a "waist" at $c<d<\sqrt{2}$. At $d<c$ it may transfer to two ovals, which can be interpreted as two atoms of the molecule separated by large distance. The results of calculations in the case of Cassinian oval do not noticeably differ from those in the case of ellipsoidal model.

In Table 5, the values of calculated evaporation heat are presented obtained by using the data on molecule volumes and linear dimensions given above.

As one can see from Table 5, employment of the results obtained in the present work gives, generally, better agreement between the calculated and experimental enthalpy of evaporation.

\section{Conclusions}

(1) The evaporation heat is determined by expression (9):

$$
\lambda=R T \ln \left(1+\frac{\Delta V}{V_{\mathrm{fL}}}\right)+P \Delta V+\frac{\sigma V_{a}}{\alpha r_{a}} .
$$

(2) At a critical point the thermodynamic parameters should satisfy relationship (14)

$$
\left(\frac{d P}{d T}\right)_{c}=\frac{R}{V_{\mathrm{fLc}}}+\frac{P_{c}}{T_{c}} .
$$

(3) The effective volumes of atoms (molecules, ions) are calculated from exact formula (15)

$$
V_{0}=\frac{1}{N_{A}}\left(V_{c}-\frac{R}{(d P / d T)_{c}-P_{c} / T_{c}}\right) .
$$

The volume calculation accuracy is only determined by the measurement accuracy for the thermodynamic parameters at the critical point. By using geometrical models for atoms (molecules, ions) one can determine their linear parameters as well.

(4) Rapid development of nanotechnology necessitates accurate determination of geometrical parameters for atoms (molecules, ions), which can be made by means of the method suggested. 


\section{References}

[1] R. C. Reid, J. M. Praunitz, and T. K. Sherwood, The Properties of Gases and Liquids, McGraw-Hill, 1977.

[2] L. D. Landau and E. M. Lifshits, Statistical Physics, ONTI, Moscow, Russia, 1938.

[3] L. D. Landau and E. M. Lifshits, Statistical Physics, vol. 1, Nauka, Moscow, Russia, 1976.

[4] I. Prigogine and D. Kondepudi, Modern Thermodynamics, Wiley, New York, NY, USA, 1999.

[5] J. Black, Lectures on the Elements of Chemistry, vol. 1, 1803.

[6] A. M. Prokhorov, Ed., Physical Encyclopaedia, Bol'shaya Sovetskaya Entsiklopediya, Moscow, Russia, 1998.

[7] R. C. Reid, J. M. Praunitz, and D. E. Poling, The Properties of Gases and Liquids, McGraw-Hill, New York, NY, USA, 1987.

[8] A. A. Sobko, "Calculating molar heat of evaporation," Doklady Physics, vol. 417, p. 326, 2007.

[9] A. A. Sobko, "Calculating molar heat of fusion," Doklady Physics, vol. 477, p. 325, 2006.

[10] K. Huang, Statistical Mechanics, John Wiley \& Sons, London, UK, 1963.

[11] N. B. Vagraftik, Handbook on Thermo-Physical Properites for Gases and Liquids, Nauka, Moscow, Russia, 1972.

[12] J. Emsley, The Elements, Clarendon Press, Oxford, UK, 1991.

[13] A. A. Revdel and A. M. Ponomareva, Brief Handbook on Physical-Chemical Parameters, Khimiya, Leningrad, Russia, 1983.

[14] A. A. Sobko, "Thermodynamic substantiation of heuristic expressions for the heat of transition of first-order phase transformations," Doklady Physics, vol. 412, p. 328, 2007.

[15] M. A. Anisimov, V. A. Rabinovich, and V. V. Sychev, Thermodynamics of Critical States for Particular Substances, Energoatomizdat, Moscow, Russia, 1990.

[16] G. V. Samoilov, Ed., Element Properties, Metallurgiya, Moscow, Russia, 1976.

[17] P. K. Rashevskii, Higher Algebra, Nauka, Moscow, Russia, 1965. 

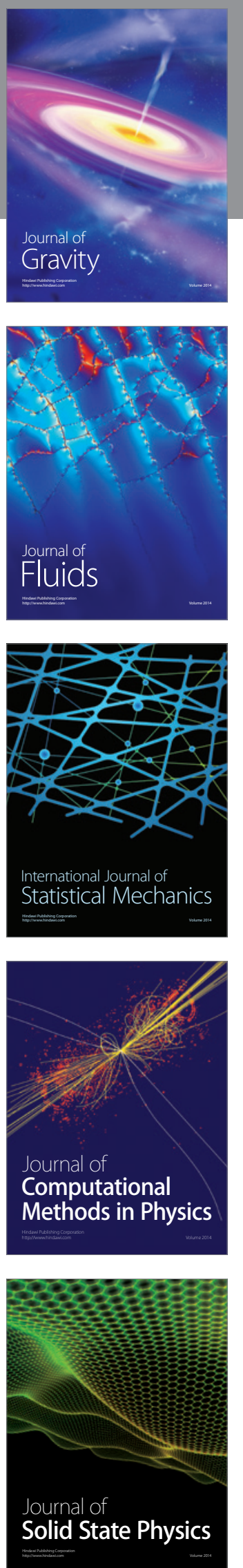

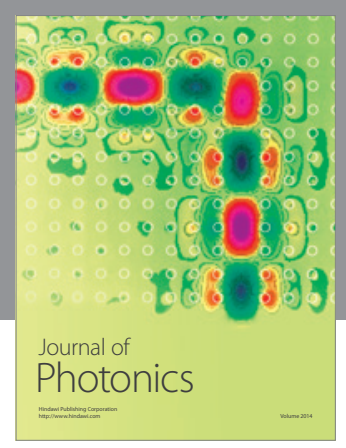

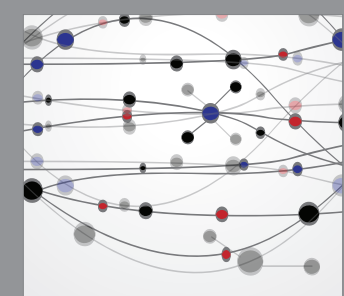

The Scientific World Journal
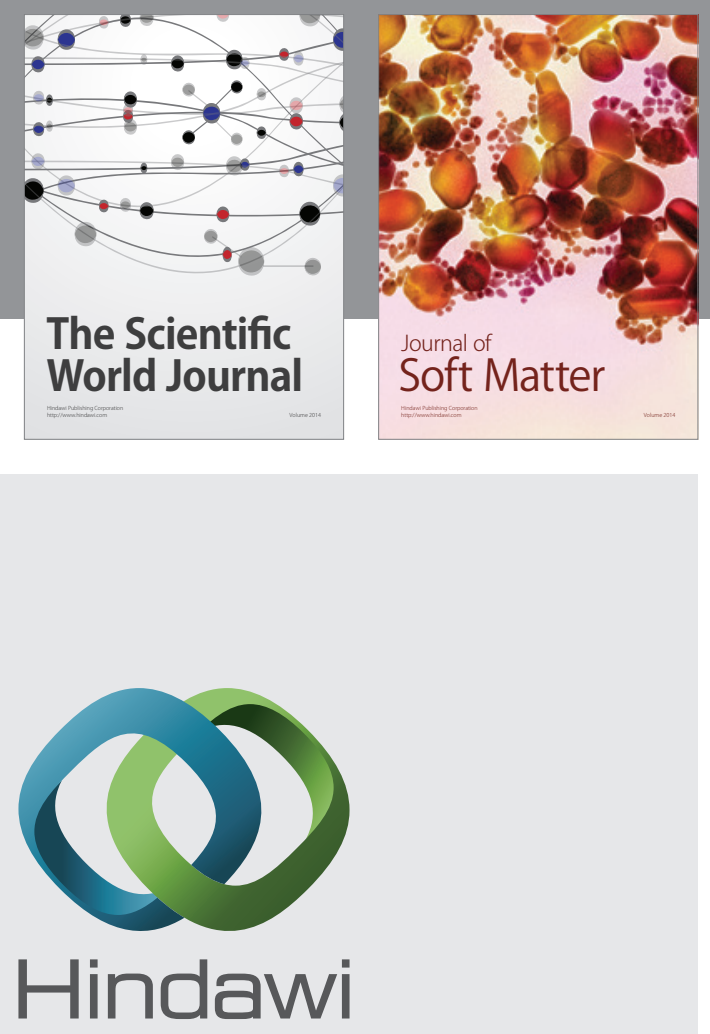

Submit your manuscripts at

http://www.hindawi.com
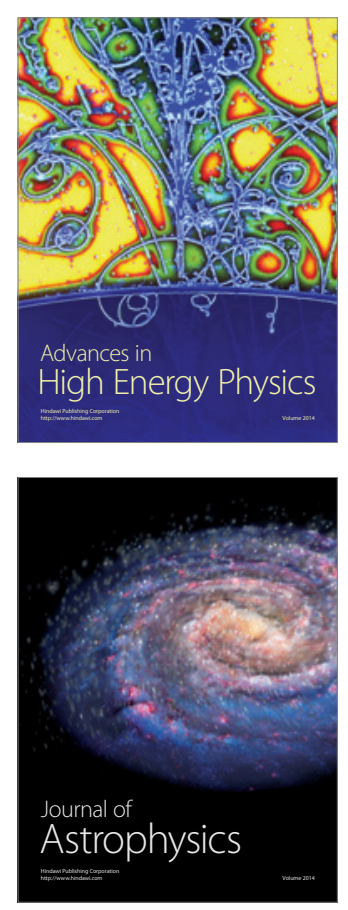
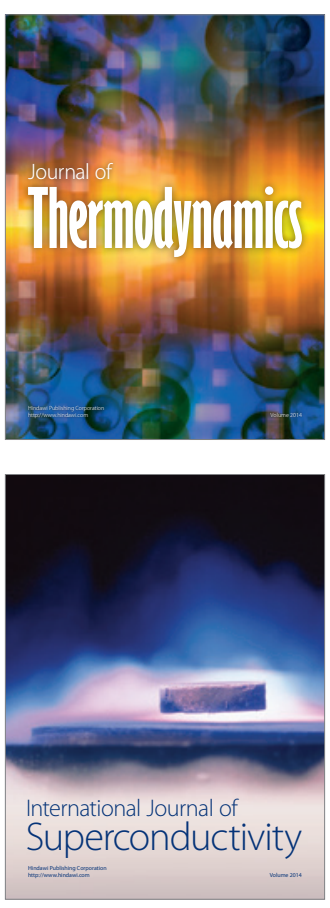
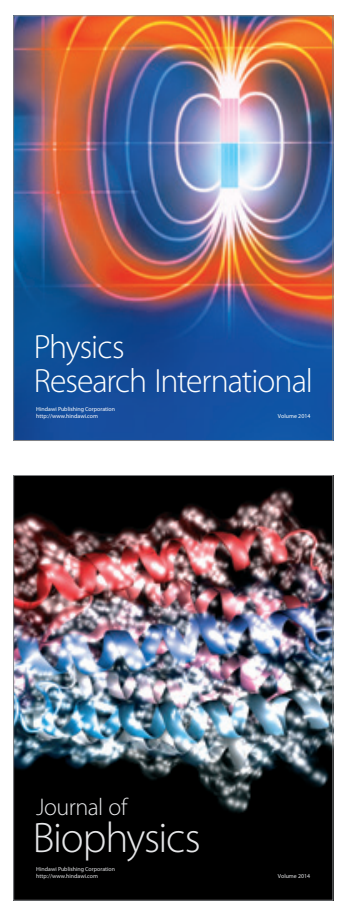
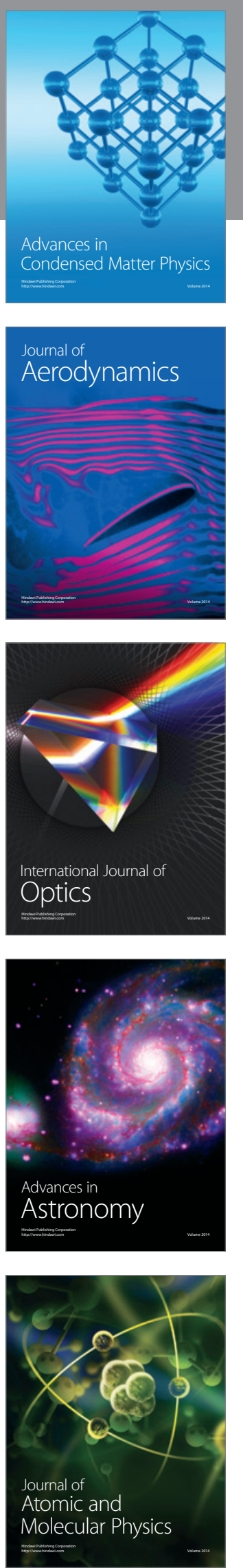